\title{
INFLUÊNCIA DA GEOMETRIA DOS DENTES DAS LÂMINAS DE SERRA DE FITA NA QUALIDADE E PRODUTIVIDADE DA MADEIRA SERRADA
}

\author{
Jair Figueiredo do Carmoํㅡㄹ João Vicente de Figueiredo Latorraca ${ }^{2}$, Márcio Pereira da Rocha ${ }^{3}$, \\ Edvá Oliveira Brito ${ }^{2}$ \\ ${ }^{1}$ Eng. Florestal, M.Sc., UFMS, Sinop, MT, Brasil - carmo.jair@gmail.com \\ ${ }^{2}$ Eng. Florestal, Dr., Depto. de Produtos Florestais, UFRRJ, Seropédica, RJ, Brasil - latorraca@hotmail.com; edva@ufrrj.br \\ ${ }^{3}$ Eng. Florestal, Dr., Depto. de Engenharia e Tecnologia Florestal, UFPR, Curitiba, PR, Brasil - mprocha@ufpr.br
}

Recebido para publicação: 15/09/2012 - Aceito para publicação: 09/01/2014

\begin{abstract}
Resumo
Este trabalho teve como objetivo avaliar o comportamento de dois modelos de dentes de lâmina de serra fita, durante o processamento mecânico de toras de Erisma uncinatum (cedrinho) e Dipteryx odorata (cumaru). A pesquisa levou em consideração aspectos qualitativos e quantitativos avaliados durante o desdobro de toras das duas espécies utilizando-se dois modelos de dentes de serra, modelo tradicional e modelo Senai. As variáveis que definiram a qualidade e a produtividade da madeira serrada, para ambos os modelos de dentes, foram, respectivamente, o desvio de corte e a velocidade de avanço do carro porta-tora. Os resultados mostraram que, para o modelo de dente denominado SENAI (MS), ganhos consideráveis em produtividade foram obtidos apenas para a espécie Erisma uncinatum. Essa vantagem não foi observada quando as toras processadas foram da espécie Dipteryx odorata, devido à consequente redução da velocidade de avanço. Em termos qualitativos, os ganhos obtidos com o modelo proposto foram observados para as duas espécies de madeira processada, com maior ênfase para madeira de cedrinho. Considerando esses resultados, o modelo Senai (MS) foi o mais recomendado para as condições de desdobro empregado, uma vez ter demonstrado ser o mais efetivo em termos de rendimento e, especialmente, qualidade de corte.

Palavra-chave: Processamento mecânico de toras; ângulos de corte; velocidade de avanço.
\end{abstract}

\begin{abstract}
Influence of geometry of the band-saw blades teeth in lumber quality and productivity. This research aimed to evaluate the behavior of two tooth models of band-saw blades, during log mechanical processing of Erisma uncinatum (cedrinho) and Dipteryx odorata (cumuru) using two types of sawtooth models: traditional model and Senai model. The research focused qualitative and quantitative aspects, evaluated during the sawing of the two species. The variables that define sawn wood quality and productivity, for both tooth models, were, respectively, the cut deviation, and the advancement velocity of $\log$ porter car. The results revealed that the "SENAI (MS)" tooth model has considerable productivity gains, only for de Erisma uncinatum specie. These advantages were not observed for the Dipteryx odorata species processed logs, due to consequent advancement velocity reduction. In qualitative terms, gains with the considered model could be observed for the two processed species, with emphasis for the medium density species. Considering the results, the Senai model (MS) was the most recommended for the applied sawing conditions, once the results demonstrated more qualitative effectiveness.

Keywords: Mechanical processing of logs; cutting angles; feed rate.
\end{abstract}

\section{INTRODUÇÃO}

De acordo com a Organização das Nações Unidas para Alimentação e Agricultura (FAO) (2009), a região da América Latina e Caribe, formada por 47 países, detém 22\% da superfície florestal mundial, 14\% da superfície de terras do globo e $7 \%$ da população do mundo. Nessa região se encontra o maior bloco contínuo de floresta pluvial tropical do mundo (Floresta Amazônica). Os desmatamentos nos trópicos e a 
degradação das florestas em muitos lugares do planeta, em especial nessa região, afetam negativamente a disponibilidade de bens e serviços florestais.

Esses dados são preocupantes, pois demonstram a fragilidade do setor florestal no Brasil frente a pressões ambientalistas internacionais, que cada vez mais questionam a necessidade do uso de nossas riquezas florestais, principalmente pela grande quantidade de desperdício da matéria-prima durante o processo de primeira transformação da madeira serrada.

O processamento primário se coloca como a principal e a primeira atividade de melhoria no setor, em que as serras de fita, especialmente as verticais, são as máquinas mais empregadas. Esse equipamento representa a maior parte do mercado nacional de serras para corte da madeira devido a sua robustez, facilidade de automação no processo produtivo e grande versatilidade, por permitir variações diamétricas durante o processo de desdobro. Segundo informações contidas no documento Junta Del Acuerdo de Cartagena (1992), as serras de fita são geralmente classificadas em função do diâmetro dos volantes das mesmas, podendo variar entre 1,00 até $3,30 \mathrm{~m}$.

De acordo com Zabot e Fernandez (2008), apesar de ser reconhecido como um setor consideravelmente forte, a exploração basicamente extrativista, com, praticamente, nenhum nível de industrialização da atividade, não permitiu que o setor se desenvolvesse ao longo dos anos, o que resulta hoje em uma gama de entraves que levam o setor a tornar-se menos atrativo ou lucrativo.

A afirmativa de Higuchi (2004) de que "os recursos naturais da Amazônia são superexplorados e subutilizados" ainda é bastante atual e apropriada para essa região, fazendo com que o desperdício gerado pelo desdobro incorreto da madeira se torne um fator preocupante e que merece maior atenção.

O fator de maior importância visando melhorias qualitativas na madeira serrada reside na afiação das lâminas, que compreende os seguintes aspectos: modelagem dos dentes da lâmina de serra de fita, quando se define a geometria dos dentes que serão utilizados; recalque e/ou estelitagem dos dentes da lâmina de serra de fita; igualização e/ou retífica lateral dos dentes da lâmina de serra de fita e afiação propriamente dita.

Atualmente, técnicas modernas de afiação permitem variar, com alguma facilidade, ângulos, altura e passo dos dentes. Permitem ainda a afiação automática de lâminas com passo variado que, segundo Sandvik (1999) e Armstrong (2005), reduzem a vibração harmônica e as consequentes ondulações na madeira.

De modo geral, no Brasil, diversos perfis de dentes são utilizados para o desdobro de madeira, independentemente da espécie. Normalmente se varia apenas a altura do dente e, em poucos casos, o ângulo de saída de cavaco $(\gamma)$.

Alguns fatores afetam o corte da madeira, e durante o processo de corte são desenvolvidas forças de direção e intensidade variáveis. As principais forças envolvidas no corte da madeira são a força paralela e a força normal. A força paralela é aquela que ocorre no sentido da direção do corte, enquanto que a força normal é aquela que ocorre em sentido perpendicular à direção do corte e, em geral, é sempre menor que a força paralela. As forças envolvidas durante o corte da madeira podem ser afetadas por fatores relacionados à própria madeira e às condições de corte (CENTRE TECHNIQUE DU BOIS ET DE L'AMEUBLEMENT (CTBA) 2001).

A velocidade de corte tem sua importância no processo e, dentro dos limites geralmente utilizados em serrarias $(\leq 45 \mathrm{~m} / \mathrm{s})$, não tem influência se a espessura de cavaco é satisfatória (CTBA, 2001). É importante lembrar que a velocidade de corte é um fator determinante da espessura de cavaco e pode exercer uma influência decisiva sobre o desgaste dos dentes da serra, devido à elevação da temperatura da ponta dos dentes durante o corte.

De acordo com Sales (1990), o esforço de corte aumenta linearmente com a espessura de cavaco para espessuras compreendidas entre 0,2 e $2,8 \mathrm{~mm}$. O limite inferior é fixado pela zona de bom corte, enquanto que o limite superior depende da resistência mecânica do dente, da lâmina e dos efeitos aplicados da massa volumétrica, do teor de umidade da madeira etc.

O mesmo autor também constatou a possibilidade de se obter um bom corte da madeira trabalhando-se dentro de certos limites de espessura de cavaco e de características da ferramenta de corte (lâmina), sendo esse intervalo denominado de zona de bom corte.

As condições de corte dependem de inúmeros parâmetros e em particular da geometria da ferramenta (CTBA, 2001). Toda ferramenta de corte é definida por três ângulos principais: ângulo de saída de cavaco, ângulo da ponta do dente e ângulo de incidência, cuja soma totaliza $90^{\circ}$. 
Carmo et al. (2012) encontraram, em estudo realizado durante o desdobro do cedrinho (Erisma uncinatum Warm.), que as maiores variações na espessura das peças desdobradas ocorreu em função da geometria dos dentes da lâmina da serra de fita empregada pela serraria. Nesse estudo, as alturas de corte avaliadas variaram de 34 a 40 centímetros para as quatro classes diamétricas avaliadas.

Os dois ângulos que mais influenciam no esforço de corte são o de incidência e o de saída de cavaco (CTBA, 2001).

De acordo com Anquetil et al. (1996), o passo, assim como a altura do dente, tem por função essencial contribuir com a boa evacuação dos cavacos produzidos no processo de serragem.

O que se busca com as modificações na geometria dos dentes da lâmina é o aumento da área útil da garganta do dente, o que é conseguido através do aumento da profundidade do dente, da espessura da lâmina, do passo do dente e de mudanças dos ângulos. Com essas alterações pode-se obter uma geometria ideal relativa a cada máquina de desdobro de toras.

Vidaurre et al. (2008), estudando o efeito da geometria dos dentes no desdobro do eucalipto, indicaram que a variação do passo do dente ocasiona maior desvio de corte.

Dalois (1977) sugere que, para aumentar a área utilizável da garganta do dente, é necessário, obrigatoriamente, aumentar a profundidade do dente e, por consequência, aumentar muitas vezes também a espessura da lâmina da serra de fita, para que ela resista à tensão mínima necessária durante o corte.

Assim sendo, este trabalho teve como objetivo principal avaliar o comportamento de dois modelos de dentes de lâminas de serras de fita, alterando a geometria do dente de uma das lâminas, durante o processamento mecânico primário de toras de duas espécies da Amazônia brasileira, cumaru e cedrinho, respectivamente de alta e média massa específica, quanto aos aspectos da qualidade e produtividade da madeira serrada.

\section{MATERIAL E MÉTODOS}

O experimento foi realizado na empresa Comércio e Indústria de Madeiras Ltda. (COIMAL), localizada no município de Sinop, no norte de Mato Grosso, distante $500 \mathrm{~km}$ da capital do estado. A empresa trabalha com mercados interno e externo e apresenta boa estrutura no processo de transformação da madeira com vistas à agregação de valor ao seu produto final.

\section{Modelos de dentes das lâminas de serra de fita}

Como referência, utilizou-se o modelo de dente mais usualmente usado pelas indústrias madeireiras no norte de Mato Grosso, sendo doravante denominado de modelo tradicional (MT). O outro modelo analisado foi o desenvolvido pelo Serviço Nacional de Aprendizagem Industrial (SENAI), unidade de Sinop, MT. Esse modelo será doravante chamado de modelo SENAI (MS), conforme adotado pelos técnicos da unidade do SENAI em Sinop, MT.

Para identificação do modelo de dente tradicionalmente utilizado pelas indústrias madeireiras do norte de Mato Grosso, utilizou-se como banco de dados os relatórios de atendimento em assessoria técnica realizados pelo SENAI - Unidade de Negócios de Sinop, MT. Esses relatórios apresentam informações importantes em relação ao modelo de dente e os ângulos mais comumente utilizados pelas serrarias, possibilitando identificar facilmente o modelo de dente mais utilizado com suas respectivas características angulares.

Para a identificação do modelo de dente mais adequado para a máquina de serra de fita da empresa, foram levados em consideração alguns aspectos peculiares da empresa, tais como modelo da máquina de afiar da empresa, diâmetro dos volantes da máquina de serra de fita, potência do motor e RPM do equipamento. O modelo de dente MS é apresentado na figura 1.

Um aspecto muito importante para definir as características de uma boa geometria do dente está relacionado ao passo do dente (distância entre as pontas dos dentes da lâmina de serra de fita). No Brasil, padronizou-se esse valor para todos os tamanhos de serra de fita, e, a princípio, não se tem informação do motivo que levou a essa padronização.

\section{Classificação e processamento mecânico das toras}

Foram selecionadas 91 toras, sendo 45 da espécie cedrinho (Erisma uncinatum) e 46 de cumaru (Dipteryx odorata). A seleção das mesmas obedeceu a alguns critérios de qualidade indicados por Zenid (1990).

As toras foram desdobradas em um engenho de serra de fita vertical, o qual apresentava boas condições de uso. O sistema de corte utilizado foi o de máximo aproveitamento de tábuas tangenciais, de 
acordo com Tuset e Duran (1979). Durante a coleta dos dados para o referente estudo, a empresa estava trabalhando com espessura nominal de $59 \mathrm{~mm}$ para as peças produzidas com a espécie cedrinho e espessura nominal de $50 \mathrm{~mm}$ para as peças produzidas com a espécie cumaru.

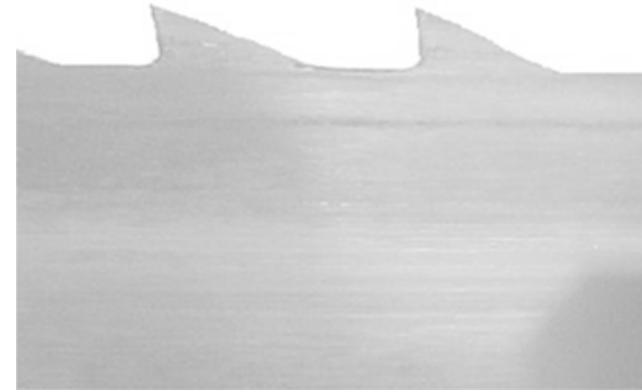

Modelo de Dente MT

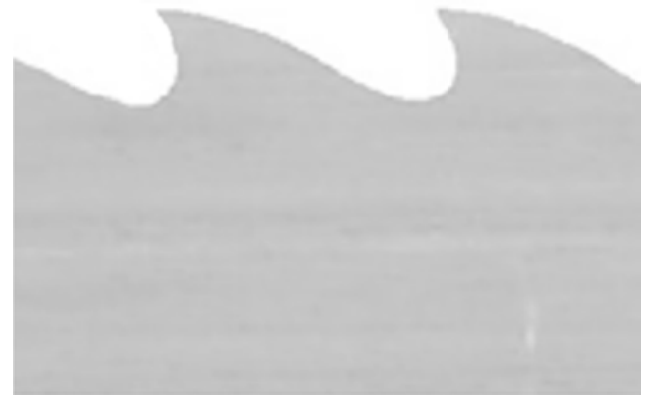

Modelo de Dente MS

Figura 1. Modelos de dentes avaliados.

Figure 1. Models of teeth evaluated.

\section{Variáveis do processo}

Os valores apresentados na tabela 1 foram também utilizados para determinar a espessura de corte $(\mathrm{t})$, a partir da velocidade média de avanço obtida durante as mensurações junto ao carro porta-toras. A equação 1 foi utilizada para determinar a espessura de corte em trabalho.

$$
t=\frac{V a \cdot p}{60 \cdot V c}(\mathrm{~mm})
$$

Em que: $V a=$ velocidade de avanço $(\mathrm{m} / \mathrm{min})$;

$t=$ espessura de corte por dente $(\mathrm{mm})$;

$V c=$ velocidade de corte $(\mathrm{m} / \mathrm{seg})$

$p=$ passo do dente $(\mathrm{mm})$.

Tabela 1. Variáveis do modelo MT e MS.

Table 1. Variables of the model MT and MS.

\begin{tabular}{lcc}
\hline \multirow{2}{*}{ Variável } & \multicolumn{2}{c}{ Modelo de dente } \\
\cline { 2 - 3 } & MT & MS \\
\hline Passo do dente $(\mathrm{mm})$ & 45 & 50 \\
Ângulo de saída de cavaco (graus) & 15 & 20 \\
Ângulo da ponta do dente (graus) & 54 & 55 \\
Ângulo incidência (graus) & 21 & 15 \\
Altura do dente para desdobro de Erisma uncinatum $(\mathrm{mm})$ & 14,8 & 15 \\
Altura do dente para desdobro de Dipteryx odorata $(\mathrm{mm})$ & 12,5 & 13,5 \\
\hline
\end{tabular}

A velocidade de corte (VC) foi dada pela equação 2, conforme descrita a seguir. Os valores do diâmetro do volante e sua rotação foram coletados in loco.

$$
V c=\frac{\pi . \phi \text { do volante. } R \text { RM do volante }}{60}(\mathrm{~m} / \mathrm{seg})
$$

\section{Classificação das toras}

A seleção das toras obedeceu a alguns critérios de qualidade indicados por Zenid (1990), a saber:

- formas, compreendendo conicidade, encurvamento, achatamentos, sapopemas e calombos ("humps");

- defeitos, compreendendo nós e protuberâncias, pequenos defeitos na superfície, rachas, rachas anelares, centro anormal e inclinação da grã; e 
- deteriorações, compreendendo furos pequenos de insetos e descoloração, furos grandes de insetos e furos de teredo, podridão no centro da tora e podridão fora do centro.

A regra utilizada para classificação geral das toras foi a do Instituto Brasileiro de Desenvolvimento Florestal (IBDF) (1984).

\section{Avaliação da qualidade e produtividade da madeira serrada}

O efeito da utilização dos dois modelos de dentes no processamento mecânico das duas espécies de madeira foi avaliado por meio do estudo da velocidade de avanço da tora e da variação na espessura ao longo do comprimento de cada prancha produzida, para expressar a variação do corte, uma vez que, segundo Vidaurre et al. (2008), a variação dimensional das peças serradas pode ser afetada pela geometria dos dentes da serra, o que acarretará, também, menores rendimentos. Essas duas variáveis permitiram avaliar os aspectos qualitativos e quantitativos, ou seja, a produtividade do processamento e a qualidade das peças (pranchas) produzidas.

A coleta dos dados da velocidade de avanço foi efetuada utilizando-se cronômetro digital. A velocidade de avanço (VA) é a relação entre o comprimento da tora e o tempo de deslocamento dessa tora em relação à lâmina da serra de fita (Equação 3).

$$
V a=\frac{\text { comprimento da prancha }}{\text { tempo de processamento de cada prancha }}(\mathrm{m} / \mathrm{min})
$$

Concomitantemente, durante a coleta da velocidade de avanço, computavam-se, também, as alturas de corte de cada prancha, para se determinar a altura média de corte por tora.

\section{Qualidade - desvio de corte}

Todas as pranchas obtidas foram medidas em espessura, após serem alinhadas e destopadas, respectivamente, em serras circular canteadeira (ou refiladeira) e destopadeira pendular.

A análise de qualidade da madeira serrada foi efetuada trabalhando-se com valores de variabilidade em espessura dentro da peça e entre peças, visando a determinação da variabilidade total, conforme a equação 6 .

Em cada prancha foram tomadas oito medidas de espessura, sendo quatro de cada lado da prancha. Para a determinação da variação de corte dentro e entre pranchas, foram utilizadas as equações 4 e 5.

$$
S w=\sqrt{\frac{\sum S^{2}}{N}}
$$

Em que: $S w=$ variabilidade dentro da prancha;

$\mathrm{S}^{2}=$ média das variâncias de todas as pranchas;

$N=$ número de amostras (pranchas).

$$
S b=\sqrt{\frac{s_{x}^{2}-\frac{S w^{2}}{n}}{n}}
$$

Em que: $S b=$ variabilidade entre pranchas;

$S \frac{2}{x}=$ desvio padrão dos valores médios para cada prancha;

$n=$ número de medições por peça.

$$
S T=\sqrt{(S w)^{2}+(S b)^{2}}
$$

Em que: $S T=$ variabilidade total. 


\section{Análises dos resultados}

O delineamento experimental foi inteiramente casualizado ao nível de $95 \%$ de probabilidade, com duas espécies e dois modelos de dentes de lâminas de serra de fita, totalizando 4 tratamentos. Dessa forma, testaram-se os dois modelos de dente de lâmina de serra de fita durante o desdobro das toras das duas espécies selecionadas, Erisma uncinatum $\left(0,59 \mathrm{~g} / \mathrm{cm}^{3}\right)$, vulgarmente conhecida como cedrinho, e Dipteryx odorata $\left(1,09 \mathrm{~g} / \mathrm{cm}^{3}\right)$, vulgarmente conhecida como cumaru.

\section{RESULTADOS E DISCUSSÃO}

\section{Classificação das toras}

Na tabela 2 são apresentadas as classes de qualidade das toras para as duas espécies avaliadas. Para a espécie E. uncinatum, $40 \%$ das toras avaliadas eram da classe I, enquanto que para a espécie $D$. odorata o maior percentual de qualidade ocorreu para a classe II, com $41,3 \%$.

Tabela 2. Classificação das toras avaliadas.

Table 2. Classification of logs.

\begin{tabular}{lcccc}
\hline \multirow{2}{*}{ Espécie } & \multirow{2}{*}{ Número de toras } & \multicolumn{3}{c}{ Número de toras por classe de qualidade } \\
\cline { 3 - 5 } & & Classe I & Classe I/II & Classe II \\
\hline E. uncinatum & 45 & 18 & 16 & 11 \\
D. odorata & 46 & 17 & 10 & 19 \\
\hline
\end{tabular}

\section{Altura de corte e espessura do cavaco}

Na tabela 3, observam-se os valores médios obtidos da altura de corte e espessura de corte para os dois modelos de dentes utilizados no processamento das duas espécies. A espessura média do corte obtida nos tratamentos mostrou uma diferença mais pronunciada somente para a espécie E. uncinatum, cujos valores a posicionam no limite inferior da zona de bom corte, denotando uma possível melhoria no padrão de qualidade da madeira serrada. A zona de bom corte definida por Sales (1990) está compreendida entre os valores de espessura de corte de $0,5 \mathrm{a}, 1,5 \mathrm{~mm}$. Para a espécie $D$. odorata, as médias foram praticamente as mesmas e, mesmo para o modelo MS, a espessura do cavaco não atingiu a zona de bom corte.

Tabela 3. Valores médios de altura e espessura de corte para os dois modelos de dentes dentro de cada espécie.

Table 3. Mean values of height and thickness of cut for the two models of teeth in each species.

\begin{tabular}{lcccc}
\hline \multirow{2}{*}{ Variável } & \multicolumn{2}{c}{ Erisma uncinatum } & \multicolumn{2}{c}{ Dipteryx odorata } \\
\cline { 2 - 5 } & MT & MS & MT & MS \\
\hline Hc - Altura média de corte $(\mathrm{cm})^{*}$ & $48,26 \mathrm{~A}$ & $49,16 \mathrm{~A}$ & $44,46 \mathrm{~B}$ & $45,19 \mathrm{~B}$ \\
$\mathrm{t}-$ Espessura de corte por dente $(\mathrm{mm})$ & 0,32 & $0,56^{* *}$ & 0,32 & 0,33
\end{tabular}

*Letras iguais denotam igualdade estatística ao nível de $95 \%$ de probabilidade pelo teste de média paramétrica. **Média compreendida dentro da zona de bom corte.

Na tabela 3, observa-se uma igualdade estatística das alturas médias, para ambas as espécies, nos dois modelos de dentes estudados. Essa igualdade dentro de cada espécie demonstra que essa variável não influenciou na qualidade da madeira serrada, de modo que a diferença na qualidade deveu-se exclusivamente à variável modelo de dente.

Observa-se que houve um aumento pronunciado na espessura de corte por dente, quando se utilizou o Modelo Senai (MS) para a espécie E. uncinatum, apresentando valores que a posicionam no limite inferior da zona de bom corte (limites de $0,5 \mathrm{~mm}$ a $1,5 \mathrm{~mm}$ ), denotando uma possível melhoria no padrão de qualidade da madeira serrada. Carmo et al. (2012) encontraram, para a mesma espécie e padrões de altura de corte semelhantes (34 a $49 \mathrm{~cm}$ ), espessura de cavaco e/ou corte com média de 0,61 mm, apresentando uma tendência de redução da espessura de corte com o aumento da altura de corte, sendo esta de $0,72 \mathrm{~mm}$ para alturas de corte de $34 \mathrm{~cm}$ e de $0,52 \mathrm{~mm}$ de espessura de corte para $49 \mathrm{~cm}$ de altura de corte. Na espécie D. odorata, os valores foram praticamente os mesmos e, mesmo para o modelo MS, a espessura de corte por dente não atingiu a zona de bom corte, ficando abaixo de $0,5 \mathrm{~mm}$ de espessura de 
cavaco. Como a espessura de corte é diretamente proporcional à velocidade de avanço, esses resultados podem representar uma maior produtividade com a mudança do modelo de dente da lâmina de serra.

\section{Qualidade das peças serradas}

Desvio total de corte

Analisando a figura 2, pode-se observar que o menor desvio de corte total (St) para a espécie $E$. uncinatum ocorreu durante a utilização do modelo de dente MS. O ganho de qualidade obtido com esse modelo no desdobro dessa espécie pode ser explicado pelo considerável aumento na espessura de corte por dente (Tabela 3). Carmo et al. (2012) encontraram para a espécie Erisma uncinatum um desvio de corte médio de 1,26 mm para um modelo de dente semelhante ao modelo MT deste trabalho, ficando próximo ao desvio médio apresentado na figura 2, cujo valor médio foi de 1,0 $\mathrm{mm}$. Vidaurre et al. (2008), trabalhando com eucalipto e correlacionando desvio de corte com classes diamétricas, verificou que o maior desvio de corte ocorreu na classe de menor diâmetro.
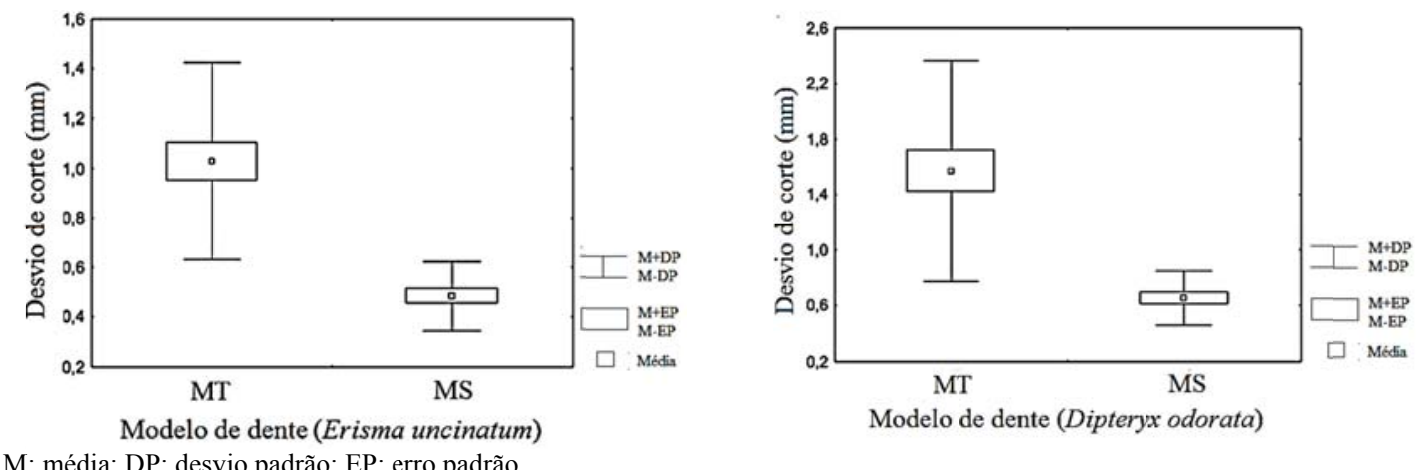

Figura 2. Desvio total de corte médio (St) no processamento da madeira de Erisma uncinatum e Dipteryx odorata para os modelos de dentes MS e MT.

Figure 2. Deviation of total average cut (St) in the processing of wood Erisma uncinatum and Dipteryx odorata for models of teeth MS and MT.

Ainda na figura 2 pode-se observar que o menor desvio de corte total $(\mathrm{St})$ para a espécie $D$. odorata ocorreu durante a utilização do modelo de dente MS. Entretanto, para o desdobro da espécie praticamente não houve diferença entre a espessura de cavaco para os dois modelos de dente, conforme constatado na tabela 2 .

Nota-se, também, por meio da figura 2, que o uso do modelo de dente MT, a exemplo do ocorrido com a espécie E. uncinatum, originou dados mais dispersos que o modelo MS. Isso gerou um desvio padrão, em relação à média, superior ao obtido pelo modelo MS.

\section{Dimensão nominal}

Com relação à dimensão nominal, observa-se nas figuras 3A e 3B, para a espécie E. uncinatum, que todas as toras apresentaram pranchas com medidas de espessura média (EM) superiores à dimensão nominal $(59 \mathrm{~mm})$ quando se utilizou o modelo de dente MT. Para o modelo de dente MS, a maioria das pranchas medidas apresentou valores superiores à dimensão nominal, porém em condições mais favoráveis que os apresentados quando da utilização do modelo de dente MT.

Para a espécie Dipteryx odorata, observa-se na figura 3C que houve, para o modelo de dente MT, uma predominância de pontos acima da dimensão nominal, estipulada em $50 \mathrm{~mm}$, com valores chegando a $52,5 \mathrm{~mm}$ de espessura. Esse aspecto demonstra uma falta de controle no processo de bitolamento das pranchas, que, mesmo sendo automática, apresentou valores superiores ao desejado. Gerwing et al. (2000), estudando dez serrarias em Paragominas, PA, encontrou uma variação total no processo de serragem de $1,8 \mathrm{~mm}$ a $8,1 \mathrm{~mm}$. Nota-se que a maioria das pranchas apresentou valores próximos à dimensão nominal para o modelo de dente MS, mas em condições mais favoráveis aos apresentados pela utilização do modelo de dente MT. Pode-se observar uma variação muito pequena em relação à dimensão nominal, principalmente se comparada com a espessura média apresentada pela utilização do modelo de dente MT. A média encontrada na sobremedida quando se utilizou o modelo de dente MS foi significativamente 
inferior à do modelo MT. Vidaurre et al. (2008) apresentaram como resultado que a utilização de passo variado, de modo geral, foi o que ocasionou maiores desvios de corte. Cabe ressaltar que os estudos foram realizados com espécies diferentes e principalmente com alturas de cortes diferentes, sendo que a altura de corte exerce influência direta na qualidade e na produtividade do processamento mecânico da madeira.
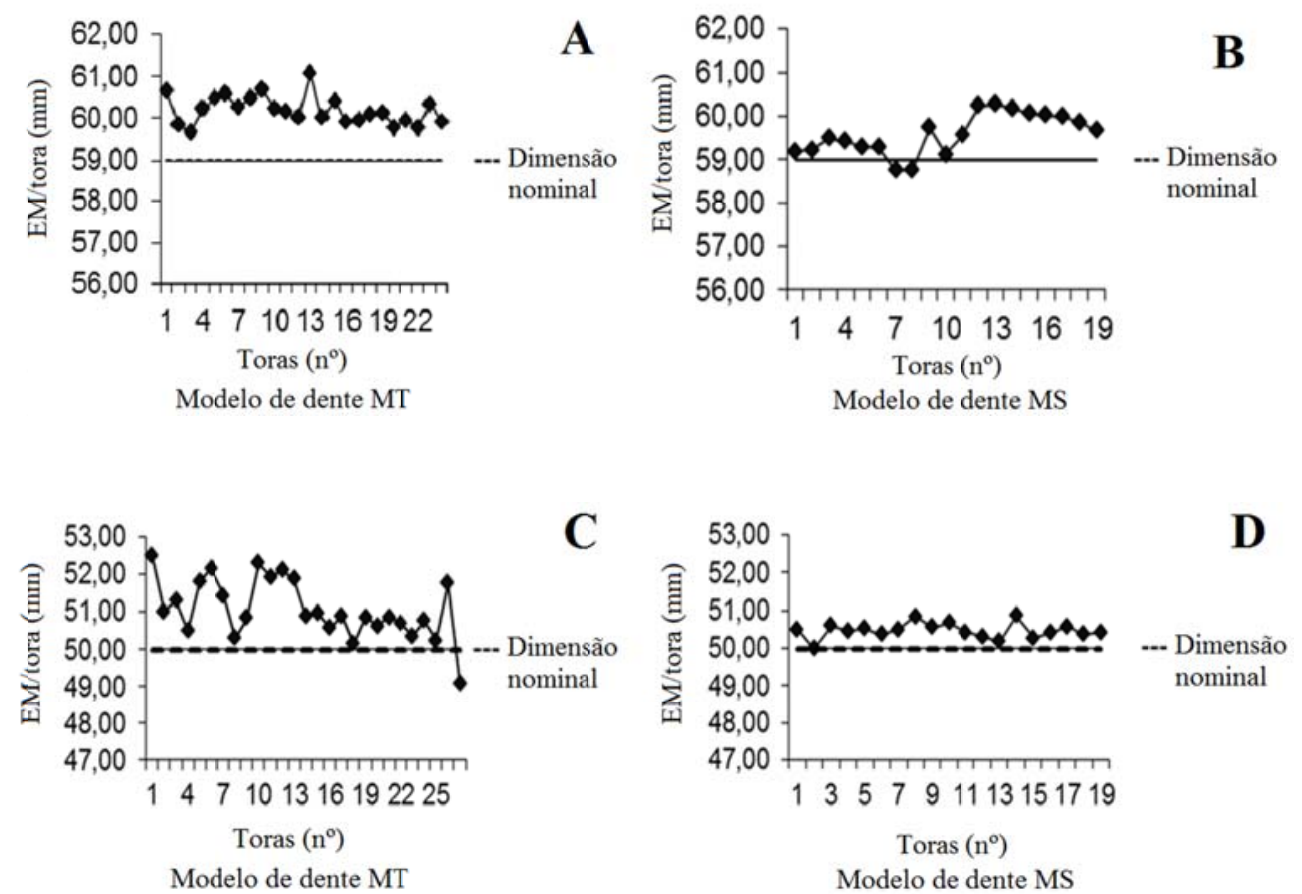

Figura 3. Espessura média (EM) das pranchas por tora de Erisma uncinatum (A e B) e Dipteryx odorata (C e D) em relação à dimensão nominal para os modelos de dentes MT e MS.

Figure 3. Average thickness (EM) of boards per log of Erisma uncinatum (A e B) and Dipteryx odorata (C e D) to the size goal for the models of teeth MS and MT.

\section{Produtividade}

Analisando a figura 4, pode-se observar que a maior velocidade de avanço (Va) durante o desdobro da espécie E. uncinatum ocorreu durante a utilização do modelo de dente MS. A média de velocidade de avanço encontrada quando se utilizou o modelo de dente MS foi maior que a do modelo MT, considerando as médias das alturas de corte coletadas durante as medições cronometradas dos avanços. A média da velocidade de avanço no desdobro do cedrinho utilizando o modelo de dente MT foi de $11,79 \mathrm{~m} / \mathrm{min}$ para uma altura de corte média de $47,28 \mathrm{~cm}$, enquanto que para o modelo de dente MS os valores médios de velocidade de avanço foram de $18,62 \mathrm{~m} / \mathrm{min}$ para uma média de altura de corte de 49,18 cm. Carmo et al. (2012), trabalhando com a espécie cedrinho, encontraram uma velocidade média de avanço de 24,72 $\mathrm{m} / \mathrm{min}$ para uma altura média de corte de $42 \mathrm{~cm}$.

Ainda na figura 4, a média de velocidade de avanço encontrada quando se utillizou o modelo de dente MS foi menor que a do modelo MT, considerando as médias das alturas de corte coletadas durante as medições cronometradas dos avanços. A média da velocidade de avanço no desdobro de $D$. odorata utilizando o modelo de dente MT foi de $11,79 \mathrm{~m} / \mathrm{min}$ para uma altura de corte média de 44,11 cm, enquanto que para o modelo de dente MS os valores médios de velocidade de avanço foram de 10,86 $\mathrm{m} / \mathrm{min}$ para uma média de altura de corte de $45,11 \mathrm{~cm}$.

\section{Gráficos de controle do processamento}

Análise do desvio padrão para madeira de média massa específica

Analisando-se a figura 5A, para o modelo de dente MT utilizado durante o desdobro da espécie $E$. uncinatum, observa-se que quatro pontos se encontram fora dos limites de controle, sendo 2 acima do limite superior de controle (LSC) e 2 abaixo do limite inferior de controle (LIC). Desvios acima do LSC e abaixo 
do LIC sugerem um processo fora de controle, o que pode ser atribuído a diversos fatores, como velocidade de avanço inadequada ou área da garganta do dente com capacidade insuficiente de armazenagem.
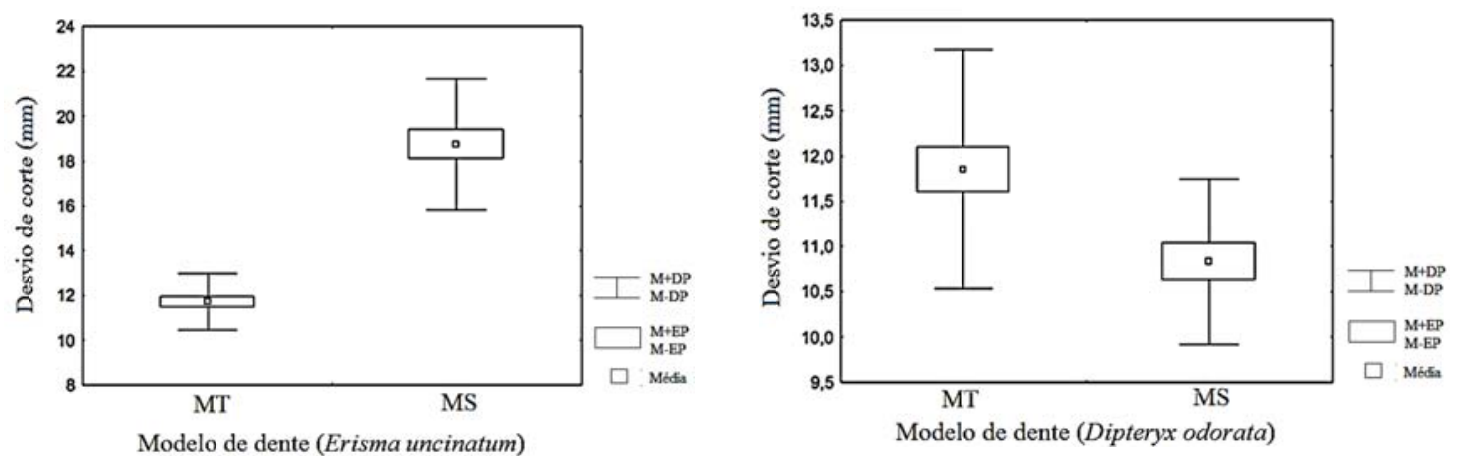

$\mathrm{M}=$ Média; $\mathrm{DP}=$ Desvio padrão; $\mathrm{EP}=$ Erro padrão

Figura 4. Velocidade de avanço média (Va) no processamento da madeira de Erisma uncinatum e Dipteryx odorata para os modelos de dentes MT e MS.

Figure 4. Average ground speed (Va) in the processing of wood Erisma uncinatum and Dipteryx odorata for models of teeth MS and MT.
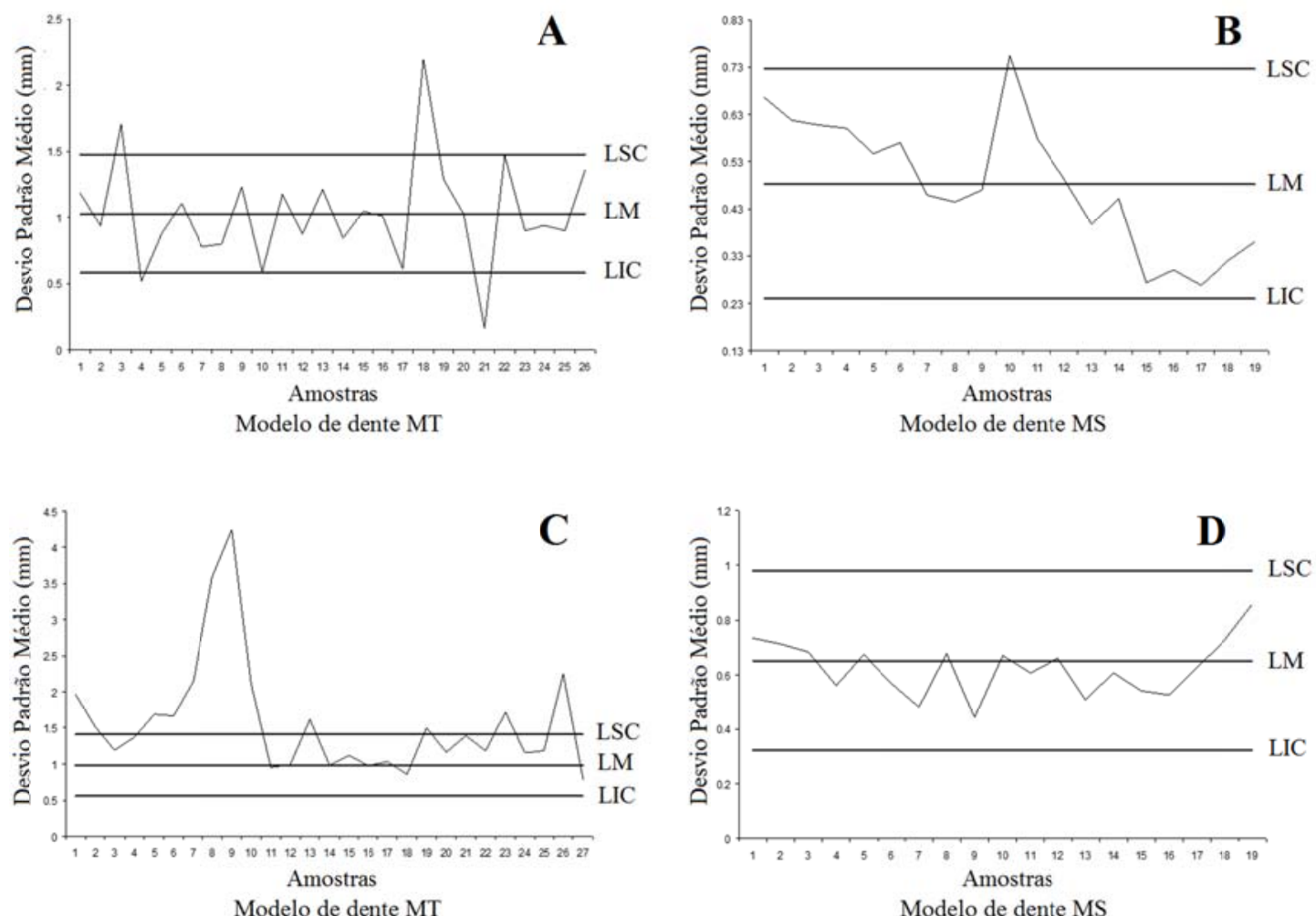

Figura 5. Carta de controle do desvio padrão do processamento da madeira de Erisma uncinatum e Dipteryx odorata utilizando os modelos de dentes MT e MS.

Figure 5. Control chart of the standard deviation of the processing of wood Erisma uncinatum (A e B) and Dipteryx odorata (C e D) using the models of teeth MT and MS. 
Já para o modelo de MS é possível observar que apenas um ponto se encontra fora dos limites de controle, sendo ele acima do limite superior de controle (LSC). Esse aspecto demonstra um processo numa condição bem mais favorável que a do gráfico do modelo MT.

No comparativo entre os dois modelos de dentes para o desdobro de madeira de média massa específica, nota-se que ocorreu uma qualidade melhor da madeira quando se utilizou o modelo MS. Notase, também, que as amplitudes dadas por LSC e LIC foram bem menores para o modelo MS do que para o modelo MT, o que indica uma variação no processo de serragem mais admissível.

$\mathrm{O}$ gráfico de controle comprova os resultados observados nas análises comparativas entre o modelo de dente MT e MS, em que, em termos gerais, o modelo de dente MS apresentou ganhos consideráveis para o desdobro de madeira de média massa específica (E. uncinatum).

Pode-se afirmar, pelos valores apresentados para madeira de média massa específica, que alterações na geometria dos dentes da lâmina da serra de fita podem auxiliar nos padrões de qualidade e produtividade da madeira serrada.

Análise do desvio padrão para madeira de alta massa específica

Analisando-se a figura 5C, para o modelo de dente MT utilizado durante o desdobro da espécie $D$. odorata, observa-se que 10 pontos se encontram fora dos limites de controle, sendo que todos acima do Limite Superior de Controle (LSC). De acordo com Latorraca (1998), esse aspecto demonstra um processo fora de controle, pois a velocidade de avanço não está compatível com o modelo de dente utilizado.

Já para o modelo de dente MS, observa-se que todos os pontos encontram-se dentro dos limites de controle. Apesar de uma maior incidência de pontos na parte inferior do gráfico, notadamente o processo estava sob controle, demonstrando uma melhor performance do modelo MS.

\section{CONCLUSÕES}

Com base nos resultados observados neste trabalho, conclui-se que:

- No desdobro de madeira de Erisma uncinatum, o modelo de dente Senai atingiu patamares de espessura de cavaco indicados para a zona de bom corte;

- No desdobro de madeira de Dipteryx odorata, os dois modelos de dentes apresentaram valores de espessura de cavaco fora da zona considerada de bom corte, com uma ligeira melhoria para o modelo de dente Senai;

- A geometria de dente Modelo Senai apresentou redução da variação total na serragem (ST) da madeira das duas espécies estudadas;

- No desdobro do Erisma uncinatum, a geometria de dente Modelo Senai proporcionou melhor velocidade de avanço;

- A madeira de Dipteryx odorata apresentou melhores resultados de velocidade de avanço quando se utilizou o modelo de dente Tradicional;

- A geometria de dente Modelo Senai apresentou menor variação na dimensão objetivo para as duas espécies;

- Os gráficos de controle de processo indicaram um melhor padrão para o modelo de dente Senai em relação ao Tradicional, indicando a utilização de uma velocidade de avanço inapropriada e uma área de garganta do dente com capacidade insuficiente de armazenagem;

- O modelo de dente Senai foi o que demonstrou ser o mais indicado para o desdobro tanto de madeira de média massa específica (Erisma uncinatum) como para madeira de alta massa específica (Dipteryx odorata).

\section{REFERÊNCIAS}

ANQUETIL, F.; BOYER, H.; JUAN, J. Entretien et Affûtage des Outils de Coupe - CTBA - Centre Technique du Bois et de L'ameublement, 1996, $206 \mathrm{p}$.

ARMSTRONG Variable pitch tooth and gullet depths. 2005. Disponível em: $<$ http//www.armstrongblue.com/publications $>$ Acesso em: 06/04/2010. 
CARMO, J. F.; LATORRACA, J. V. F.; SILVA, C. R. P.; POUBEL, D. S.; SILVA, I. M. M.; SOUZA, W. N. Avaliação dos parâmetros de corte na qualidade da madeira serrada no desdobro do cedrinho (Erisma uncinatum Warm.). Anais do VII Simpósio de Pós-Graduação em Ciências Florestais VIÇOSA - MG, 17 a 18 de setembro de 2012.

DALOIS, C. Manuel de Sciage et D’Affûtage - C.T.F.T. - Centre Technique Forestier Tropical. 1977. $199 \mathrm{p}$.

GERWING, J.; VIDAL, E.; VERÍSSIMO, A.; UHL, C. Rendimento no Processamento de Madeiras no Estado do Pará. Série Amazônia (v. 18, p. 38), 2000. Belém: Imazon.

HIGUCHI, N. Amazônia - interesses e conflitos, Reportagens - Revista Comciência, 2004. Disponível em: <http://WWW.comciencia.br>. Acesso em: 02/2010.

INSTITUTO BRASILEIRO DE DESENVOLVIMENTO FLORESTAL (IBDF). Normas para medição e classificação de toras de madeiras de folhosas. Brasília, 1984, p. 42.

LATORRACA, J. V. F. Processamento Mecânico da Madeira. Apostila. Universidade Federal Rural do Rio de Janeiro. Rio de Janeiro. 1998. 197 p.

Junta Del Acuerdo de Cartagena - Manual del grupo andino para aserrio y afilado de sierras cintas y sierras circulares. Lima, 1992, $270 \mathrm{p}$.

MANUEL SCIERIE. Thecniques et Matériels - CTBA - Centre Technique du Bois et de L'ameublement, 2001, $397 \mathrm{p}$.

ORGANIZAÇÃO DAS NAÇÕES UNIDAS PARA ALIMENTAÇÃO E AGRICULTURA (FAO). Situación de los Bosques del Mundo, 2009. Disponível em: <http://www.fao.org>. Acesso em 20/03/2010.

SALES, C. La Scie a Ruban. Théorie et Pratique du Sciage des Bois em Grume - C.T.F.T - Centre Technique Forestier Tropical, 1990, 150 p.

SANDVIK - Manual from sandvik steel Production, use and maintenance of wood bandsaw blades. Sweden, p. 82, 1999.

TUSET, R.; DURAN, F. Manual de Maderas Comerciales, Equipos y Procesos de Utilizacion. Editorial JHemisferio Sur, Montevideo, 1979, 688 p.

VIDAURRE, G. B.; VITAL, B. R.; OLIVEIRA, J. T. S.; CARVALHO, A. M. M. L.; DELLA LUCIA, R. M.; CARNEIRO, A. C. O. Efeito da geometria dos dentes da serra de fita na produção de madeira serrada de eucalipto. Revista Árvore, v. 32, n. 5, p. 939 - 947.

ZABOT, U. C.; FERNANDEZ, G. S. Caracterização e quantificação da exploração madeireira nas cidades da região norte do Mato Grosso. Projeto Resíduos Sólidos da Madeira - Aprovado e Financiado pela FAPEMAT - Depto. de Economia - Unemat - Campus Universitário de Sinop, 2008, 8 p.

ZENID, G. J. Noções de Classificação de Toras e de Madeiras Serradas. IPT - Instituto de Pesquisas Tecnológicas, 1990. 
